\title{
Flavonols in skins of wild grapes (Vitis vinifera L., subsp. sylvestris (Gmelin) Hegi)
}

\author{
Eugenio Revilla ${ }^{1}$, Alberto Bellido ${ }^{2}$, Joaquín Yus ${ }^{1}$, Patricia Ortiz ${ }^{1}$, David Carrasco ${ }^{2}$, and Rosa A. Arroyo ${ }^{2}$ \\ ${ }^{1}$ Departamento de Química Agrícola y Bromatología. Universidad Autónoma de Madrid, 28049 Madrid, Spain \\ ${ }^{2}$ CBGP-UPM-INIA, Departamento de Biotecnología, Campus de Montegancedo, 28223 Pozuelo de Alarcón, Spain
}

\begin{abstract}
Flavonols are a group of grape phenolics that play an important role in young red wines, as they are involved in copigmentation of the flavylium form of anthocyanins. A study on the flavonol composition of grape skins in several wild grapevine genotypes from different Iberian natural populations, preserved at El Encin Germoplasm Bank, has been carried out in 2012. Flavonol glycosides contained in grape skins were determined by HPLC-DAD and HPLC-MS, through a previous phase of purification, using ion exchange chromatographic columns to retain anthocyanins and other phenolic compounds, so that flavonols do not suffer co-elution with other components, improving HPLC analysis. Thus, it was possible to separate 12 flavonol glycosides, and eight of them were successfully identified. The major flavonols were quercetin-3-O-glucoside, quercetin-3-O-glucoronide and myricetin-3-O-glucoside. The diversity and number of flavonols differed for each genotype. The total content of flavonols ranged from 25 to $350 \mathrm{mg} / \mathrm{kg}$ grapes; the richest genotype was three times richer than Tempranillo grapes, used as a reference. The most significant difference between wild genotypes and reference cultivars was that, in many cases, myricetin-3-O-glucoside or quercetin-3-Oglucuronide predominated in wild genotypes.
\end{abstract}

\section{Introduction}

Flavonols are a class of grape phenolics that play an important role in young red wines, as they are involved in copigmentation of the flavylium form of anthocyanins [1]. Moroever, flavonols present positive effects on health due to their antioxidant properties [2], and are widely distributed in dietary plants [3]. Flavonols are yellow pigments that are predominantly synthesized in grape skins [4], and their accumulation take place in response to sunlight; the studies carried out in Shiraz grapes suggest that the branch of flavonoid biosynthetic pathway leading to flavonol biosynthesis is light-dependent, in contrast to anthocyanin and flavanol biosynthesis, which are little affected by shading treatments [5].

The presence of flavonol aglycones in wines is well documented, but the flavonol composition of grapes is more complex, as they contain glycosides, that are extracted from grape skins and suffer hydrolysis by $\beta$-glucosidases in the initial steps of winemaking. Thus, the flavonol composition of grapes has been studied in deep only for a reduced number of cultivars [5-7], despite the potencial value of flavonols as chemical markers to evaluate cultivar authenticity in grapes and wines [8].

Cultivated grapevines are thought to be domesticated from wild populations of Vitis vinifera L. ssp. sylvestris (Gmelin) Hegi [9]. These small, isolated wild populations are distributed in riverbanks from the western Himalayas to Western Europe [10], and their sanitary status and morphological and genetic characteristics have been studied in recent years [11-14]. Wild grapevines present some interesting oenological characteristics, as high acidity, low $\mathrm{pH}$ and a high intensity of color $[15,16]$; these features might be used to mitigate the potential effects of global warming on grape production, using a natural resource to adapt Viticulture to the new climatic conditions.

After 2006, our research group has been examining the qualitative and quantitative anthocyanin composition of wild grape accessions preserved at El Encin Germoplasm Bank $[17,18]$. Taking into account the importance of flavonols on the copigmentation of anthocyanins in wines, it has been considered of interest to examine the presence of flavonols in wild grapes. The objectives of our study are to evaluate whether the flavonol composition of wild grapes differs from that presented by cultivated grapes, and to determine whether some wild genotypes present some genetic characters of interest related to flavonol accumulation during grape maturation.

\section{Materials and methods}

\subsection{Grape samples}

Samples of 16 genotypes of wild grapevines from different Iberian natural populations preserved at El Encin Grapevine Germoplasm Bank (IMIDRA, Alcalá de Henares, Spain), as well as grapes of Pinot noir and Tempranillo cultivars, were collected in October 2012 at optimum stage of maturation, each sample consisted of four clusters. Once in the laboratory, samples were stored at $-20^{\circ} \mathrm{C}$ until sample preparation.

\subsection{Reagents and standards}

Deionized water was purified with a Milli-Q water system (Millipore, Bedford, MA) before use. Acetonitrile of 
HPLC grade was purchased from Merck (Darmstadt, Germany). Trifluoroacetic acid of analytical reagent grade, and standards of flavonols were obtained from Sigma-Aldrich (Tres Cantos, Spain. All other chemicals (analytical-reagent grade) were obtained from Panreac (Mollet del Vallès, Spain).

\subsection{Sample preparation}

Berries were separated from clusters; then, 50 berries were randomly selected and weighed. After that, grape skins were separated from pulps and seeds, and conserved in methanol at $-20^{\circ} \mathrm{C}$. Then, grape skins were grinded in a Kinematica PCU-2 blender for 1 minute, and submitted to sequential extraction with different solvents (methanol for 16 hours at $-25^{\circ} \mathrm{C}, 80 \%$ methanol for four hours at room temperature, $50 \%$ methanol for four hours at room temperature, deionized water for 16 hours at $-25^{\circ} \mathrm{C}$, and $75 \%$ acetone for one hour at room temperature) using $25 \mathrm{~mL}$ of solvent for each extraction step, as described elsewhere [19]. When each extraction step was finished, the liquid extract was separated by centrifugation at $3500 \mathrm{rpm}$ for 20 minutes in a Rotofix 32A centrifuge, and the residue was submitted to extraction again. For each sample, all the liquid extracts were combined, and their volume was raised between 125 and $200 \mathrm{~mL}$ with methanol. Then, the extracts were stored at $4{ }^{\circ} \mathrm{C}$ prior to their analysis. Additionally, $\sim 50 \mathrm{~g}$ berries were crushed for determining sugar concentration of must by refractometry and total acidity by potentiometry, following OIV standard procedures [20].

\subsection{Isolation of flavonols}

Before HPLC analysis, flavonols were isolated from grape extracts to avoid interferences caused by anthocyanins. For this purpose, solid-phase extraction on Oasis MCX cartridges (6 mL capacity) filled with $500 \mathrm{mg}$ of adsorbent (Waters Corp., Milford, MA), containing a mixture of reverse-phase and cationic-exchanger materials, was carried out, following a procedure described previously [6]. Prior to separation, $3 \mathrm{~mL}$ grape extracts was dried in a rotary evaporator $\left(40^{\circ} \mathrm{C}\right)$ and re-solved in $3 \mathrm{~mL}$ of $0.1 \mathrm{M}$ hydrochloric acid. The prepared samples were passed through the MCX cartridges previously conditioned with methanol $(5 \mathrm{~mL})$ and water $(5 \mathrm{~mL})$. After washing with $0.1 \mathrm{M}$ hydrochloric acid $(5 \mathrm{~mL})$ and water $(5 \mathrm{~mL})$, the flavonol fraction was eluted with methanol $(3 \times 5 \mathrm{~mL})$. This fraction also contained other neutral or acidic polyphenols. Fixed anthocyanins were removed using $2 \%$ ammonia in $80 \%$ methanol $(3 \times 5 \mathrm{~mL})$, and the cationic-exchanger material was regenerated $0.52 \mathrm{M}$ hydrochloric acid in $80 \%$ methanol $(3 \times 5 \mathrm{~mL})$. Subsequent conditioning of the cartridge with methanol and water allows its reuse at least four or five more times. The eluate containing flavonols was dried in a rotary evaporator $\left(40^{\circ} \mathrm{C}\right)$ and re-solved in $1 \mathrm{~mL}$ of methanol.

\subsection{HPLC analysis of flavonols}

The flavonol profile of the skin extracts was obtained with HPLC-DAD, using a procedure based on other described previously [21] with small changes [17]. Analysis were performed with a liquid chromatograph consisting of a 600 quaternary pump, a 717 automatic injector, a
TC2 controller for a column oven, a 996 photodiode array detector, and a Millennium 32 workstation (Waters, Milford, MA). The separation was carried out using a Waters Nova-Pak C18 steel cartridge $(3.9 \times 250 \mathrm{~mm})$, filled with $5-\mu \mathrm{m}$ particles, and furnished with a Waters Sentry Nova-Pack C18 guard cartridge $(20 \times 3.9 \mathrm{~mm})$, both thermostated at $55^{\circ} \mathrm{C}$. Two mobile phases were used: water/acetonitrile (95:5) adjusted to $\mathrm{pH} 1.3$ with trifluoraectic acid (solvent $\mathrm{A}$ ), and water/acetonitrile (50:50) adjusted to $\mathrm{pH} 1.3$ with trifluoracetic acid (solvent B). Gradient elution was applied at a $0.8 \mathrm{~mL} / \mathrm{min}$ flow rate according to the following program: linear gradient from $10 \% \mathrm{~B}$ to $35 \% \mathrm{~B}$ in $30 \mathrm{~min}$, from $35 \% \mathrm{~B}$ to $50 \% \mathrm{~B}$ in $6 \mathrm{~min}$, from $50 \% \mathrm{~B}$ to $100 \% \mathrm{~B}$ in $8 \mathrm{~min}, 100 \% \mathrm{~B}$ for $3 \mathrm{~min}, 100 \%$ B to $10 \%$ B in $1 \mathrm{~min}$. Samples $(20 \mu \mathrm{L})$ were injected in triplicate. Spectra were recorded every second between 250 and $600 \mathrm{~nm}$, with a bandwidth of $1.2 \mathrm{~nm}$. Samples, standard solutions of querecetin-3- $O$-glucoside, and mobile phases were filtered before analysis through a $0.45-\mu \mathrm{m}$ pore size membrane.

The identity of the different flavonols present in samples was determined by HPLC-MS, using an 1100 HPLC system (Agilent Technologies, Santa Clara, CA) with a PDA UV-Vis detector coupled to a QTOF mass spectrometer (AB SCiex, Framingahm, MA) Chromatographic conditions were those used for HPLCDAD analysis. MS analysis were carried out in the ESI+ mode, scanning from $\mathrm{m} / \mathrm{z} 50$ to 2000 , using the following coditions: spray voltage, $5500 \mathrm{~V}$; gas pressure, $80 \mathrm{psi}$; declustering potential, $50 \mathrm{~V}$; focus potential, $210 \mathrm{~V}$; CAD, 3 psi.

Quantitative analysis of flavonols was carried out considering the surface of the different peaks, using standard solutions of quercetin-3- $O$-glucoside in the range of 20 to $100 \mathrm{mg} / \mathrm{L}$.

\section{Results and discussion}

\subsection{HPLC-MS identification of flavonols in grape skins}

Six different flavonols (FL2, FL5, FL6, FL7, FL9 and FL12) have successfully identified by HPLC-MS, and other two (FL1 and FL4) have been tentatively identified. Table 1 displays the masses of the molecular ion and of the major fragment, $\lambda_{\max }$ values in the UV-Vis region, and the identity of those compounds. Other four flavonols have been detected in some samples, but their identity has not been elucidated by HPLC-MS. Anyway, they are minor components, and their content never exceeded $12 \mathrm{mg} / \mathrm{kg}$ grapes, and are usually absent in most samples.

The six flavonols fully identified were a myricetin derivative (3-O-glucoside, $\mathrm{MyGl}$ ), three quercetin derivatives (3- $O$-glucoside, QuGl; 3- $O$-glucuronide, QuGr; 3-O-rhamnoside, QuRh), a laricitryn derivative (3-Oglucoside, LaGl) and a syringetin derivative (3-Oglucoside, SyGl). All these flavonols have identified in berries of several red grapevine cultivars $[6,7]$, and their presence in wild grapes should be expected, as wild grapes are red colored. The flavonols tentatively identified were 3-O-galactosides of myricetin (MyGal) and quercetin (QuGal), which have been previously detected in red grape skins [7]. 
Table 1. Mass spectral and UV-Vis data of flavonols identified in grape skins (*: tentatively identified).

\begin{tabular}{|l|c|c|l|c|}
\hline Compound & $\begin{array}{c}\text { Molecular ion } \\
(\boldsymbol{m} / \boldsymbol{z})\end{array}$ & $\begin{array}{c}\text { Fragment } \\
\text { ion }(\boldsymbol{m} / \mathbf{z})\end{array}$ & $\lambda_{\text {max }}$ & Identity \\
\hline FL1 & 481 & 319 & $\begin{array}{l}353,300(\mathrm{~s}), \\
260\end{array}$ & MvGal $^{*}$ \\
\hline FL2 & 481 & 319 & $\begin{array}{l}353,300(\mathrm{~s}), \\
260\end{array}$ & MvGl \\
\hline FL4 & 465 & 303 & $\begin{array}{l}352,300(\mathrm{~s}), \\
253\end{array}$ & QuGal \\
\hline FL5 & 479 & 303 & $\begin{array}{l}352,300(\mathrm{~s}), \\
255\end{array}$ & QuGr \\
\hline FL6 & 465 & 303 & $\begin{array}{l}352,300(\mathrm{~s}), \\
255\end{array}$ & QuGl \\
\hline FL7 & 495 & 333 & $\begin{array}{l}356,300(\mathrm{~s}), \\
259\end{array}$ & LaGl \\
\hline FL9 & 449 & 303 & 345,264 & QuRh \\
\hline FL12 & 509 & 347 & $\begin{array}{l}358,305(\mathrm{~s}), \\
264\end{array}$ & SyGl \\
\hline
\end{tabular}

\subsection{Quantitative analysis of flavonols in grape skins}

Tables 2 and 3 summarize the quantitative data obtained after the examination of chromatograms obtained for the extracts of berry skins of 16 genotypes of wild grapes, and those obtained for reference grape cultivars (Pinot noir and Tempranillo). Total content of flavonols, taking into account the content of the four flavonols that have not been fully identified, ranged from $26.9 \mathrm{mg} / \mathrm{kg}$ grapes (genotype CA-6.1) to $349.7 \mathrm{mg} / \mathrm{kg}$ grapes (genotype H-6.1); these results are similar to those reported previously by other authors in red grape cultivars [6,7].

The most abundant flavonols were, in most cases, the 3-O-glucosides of myricetin (MyGl) and quercetin $(\mathrm{QuGl})$, and the 3-O-glucuronide of quercetin $(\mathrm{QuGr})$; in most cases, their content was higher than $20 \mathrm{mg} / \mathrm{kg}$ grapes, and sometimes exceeded $50 \mathrm{mg} / \mathrm{kg}$ grapes. These results are similar to those obtained previously in red grape cultivars [6,7]. Among the other flavonols, their content was usually low, less than $10 \mathrm{mg} / \mathrm{kg}$ grapes; the most remarkable exception was wild genotype H-6.1, which presented a remarkable content of QuGal. Usually, QuGr (seven genotypes and cv. Pinot noir) or QuGl (four genotypes) were the most abundant flavonols, but in some cases (wild genotypes BA-1.1, CO-7.6, CR-1.3, SE-3.2 and SS-2.3, and cv. Tempranillo) the most abundant flavonol was MyGl. In these samples, the levels of other B-ring trisubstituted flavonols (3-O-glucosides of laricitrin, LaGl, and syringetin, SyGl) are usually higher than in other samples, and this fact probably is related to differences in the regulation of genes involved in the hydroxylation of B-ring by flavonoid-3',5'-hydroxylase (F3'5'H).

\section{Conclusions}

The wild grape genotypes under study contained different flavonols in skins. Among them, several derivatives of quercetin, myricetin, laricitrin and syringetin have been detected, at it could be expected in red grapes. In most cases, the most abundant flavonol in wild grapes is myricetin-3-O-glucoside or quercetin-3-O-glucuronide. In those genotypes which presented myricetin-3-Oglucoside as major flavonol, the levels of other B-ring
Table 2. Content of quercetin derivatives ( $\mathrm{mg} / \mathrm{kg}$ grapes) in wild grape genotypes and in reference cultivars.

\begin{tabular}{|l|c|c|c|c|}
\hline Sample & QuGal & QuGr & QuGl & QuRh \\
\hline BA-1.1 & nd & 15.3 & 5.8 & nd \\
\hline CA-4.1 & 2,6 & 26.2 & 22.9 & 9.6 \\
\hline CA-6.1 & nd & 10.9 & 7.5 & nd \\
\hline CA-10.3 & 2.5 & 30.0 & 30.1 & 11.2 \\
\hline CA-12.5 & 1.9 & 37.1 & 19.3 & nd \\
\hline H-2.7 & 6.2 & 22.6 & 37.0 & 2.2 \\
\hline H-4.1 & 3.5 & 50.9 & 38.5 & 3.0 \\
\hline H-6.1 & 57.0 & 100.8 & 49.3 & 12.4 \\
\hline CO-7.6 & 3.3 & 20.3 & 29.5 & 1.3 \\
\hline CR-1.3 & 1.9 & 31.0 & 25.6 & nd \\
\hline SE-3.2 & 1.7 & 34.6 & 15.3 & nd \\
\hline FR-2.1 & 3.8 & 28.8 & 38.9 & 0.8 \\
\hline LE-1.1 & 1.8 & 33.2 & 21.7 & nd \\
\hline NA-1.4bis & 4.3 & 62.8 & 38.1 & 1.3 \\
\hline S-1.9 & 7.1 & 72.9 & 48.5 & nd \\
\hline SS-2.3 & 2.2 & 39.3 & 20.9 & nd \\
\hline Pinot noir & 6.2 & 53.1 & 50.1 & 7.0 \\
\hline Tempranillo & 5.2 & 20.8 & 30.5 & 11.3 \\
\hline
\end{tabular}

nd: not detected.

Table 3. Content of myricetin, laritricin and syringetin derivatives (mg/k grapes) in wild grape genotypes and in reference cultivars.

\begin{tabular}{|l|c|c|c|c|}
\hline Sample & MyGal & MyGl & LaGl & SyGl \\
\hline BA-1.1 & 2.2 & 41.8 & 4.4 & 3.2 \\
\hline CA-4.1 & 2.2 & 22.3 & 5.4 & 4.8 \\
\hline CA-6.1 & nd & 2.5 & 0.7 & 0.8 \\
\hline CA-10.3 & 2.2 & 18.6 & 5.5 & 5.7 \\
\hline CA-12.5 & 1.7 & 15.8 & 5.2 & 6.4 \\
\hline H-2.7 & nd & 4.0 & 1.0 & 1.6 \\
\hline H-4.1 & 2.6 & 12.2 & 4.4 & 5.2 \\
\hline H-6.1 & 11.9 & 94.2 & 3.5 & 2.0 \\
\hline CO-7.6 & 4.3 & 29.6 & 2.3 & 1.1 \\
\hline CR-1.3 & 3.0 & 35.0 & 8.4 & 10.9 \\
\hline SE-3.2 & 8.3 & 62.7 & 10.4 & 6.3 \\
\hline FR-2.1 & 3.4 & 35.5 & 3.1 & 1.5 \\
\hline LE-1.1 & 2.0 & 15.8 & 5.4 & 7.8 \\
\hline NA-1.4bis & 2.7 & 24.8 & 1.0 & nd \\
\hline S-1.9 & 3.2 & 36.0 & 3.7 & 1.4 \\
\hline SS-2.3 & 9.6 & 48.0 & 11.3 & 11.7 \\
\hline Pinot noir & 5.5 & 21.1 & 9.6 & 8.8 \\
\hline Tempranillo & 7.2 & 37.2 & 6.6 & 3.6 \\
\hline
\end{tabular}

nd: not detected.

trisubstituted flavonols are usually higher than in other genotypes. This fact probably reflects differences in the regulation of genes involved in the hydroxylation of B-ring by flavonoid-3',5'-hydroxylase (F3' 5' H).

Authors acknowledge the financial support of Spain's Ministry of Economy and Competitiveness (Project RTA2014-0016-C00301, MINECO-INIA-CCAA).

\section{References}

[1] R. Boulton, Am. J. Enol. Vitic. 52, 67 (2001)

[2] S. Burda, W. Oleszek, J. Agric. Food Chem. 49, 2774 (2001)

[3] C. Manach, A. Scalbert, C. Morand, C. Remesy, L. Jimenez, Am. J. Clin. Nutr. 79, 727 (2004) 
[4] S.F. Price, P.J. Breen, M. Valladao, B.T. Watson, Am. J. Enol. Vitic. 46, 187 (1995)

[5] M.A. Downey, J.S. Harvey, S.P. Robinson, Austr. J. Grape Wine Res. 10, 54 (2005)

[6] N. Castillo-Muñoz, S. Gomez-Alonso, E. GarciaRomero, I. Hermosin-Gutierrez, J. Agric. Food Chem. 55, 992 (2007)

[7] M. Figueiredo-Gonzalez, E. Martinez-Carballo, B. Cancho-Grande, J.L. Santiago, M.C. Martinez, J. Simal-Gandara, Food Chem. 130, 9 (2012)

[8] F. Mattivi, R. Guzzon, U. Vrhovsek, M. Stefanini, R. Velasco, J. Agric. Food Chem. 54, 7692 (2006)

[9] L. Levadoux, Ann. Amelior. Plant. 6, 59 (1956)

[10] D. Zohary, M. Hopf, E. Weiss, Domestication of Plants in the Old World (Oxford University Press, Oxford, 2012)

[11] C. Arnold, F. Guillet, J.M. Gobat, Vitis, 37, 159 (1998)

[12] R. Ocete, M.A. López, M.A. Pérez, R. Tio, $R$. Las poblaciones españolas de vid silvestre (INIA, Madrid, 1999)

[13] J. Cunha, M. Teixeira-Santos, L.C. Carneiro, P. Fevereiro, J.E. Eiras-Dias, Genet Resour. Crop Evol. 56, 975 (2009)
[14] M.T. De Andres, A. Benito, G. Perez-Rivera, R. Ocete, M.A. Lopez, L. Gaforio, G. Muñoz, F. Cabello, J.M. Martinez-Zapater, R. Arroyo-Garcia, Mol. Ecol.21, 800 (2012)

[15] R. Ocete, R. Arroyo-Garcia, M.L. Morales, M. Cantos, A. Gallardo, M.A. Perez, I. Gomez, M.A. Lopez, Vitis 50, 11 (2011)

[16] R. Ocete, M. Cantos, A, Lopez-Gallardo, A. PerezTroncoso, M. Lara, O. Failla, F.J. Ferragut, J. Liñan, Caracterización y conservación del recurso fitogenético vid silvestre en Andalucía (Falcor, Sevilla, 2007)

[17] E. Revilla, D. Carrasco, A. Benito, R. Arroyo-Garcia, Am. J. Enol. Vitic. 61, 636 (2010)

[18] E. Revilla, D. Carrasco, V. Carrasco, A. Benito, R. Arroyo-Garcia, Vitis, 51, 161 (2012)

[19] E. Revilla, J.M. Ryan, G. Martin-Ortega, J. Agric. Food Chem. 46, 4592 (1998)

[20] Organization Internationale de la Vigne et du Vin, Recueil des Méthodes Internationales d'Analyse des Vins et des Mouts (OIV, Paris, 2008)

[21] E. Garcia-Beneytez, F. Cabello, E. Revilla, J. Agric. Food Chem., 51, 5622 (2003) 